\section{ESTABLECIMIENTO DE LOS PARÁMETROS OPERACIONALES ÓPTIMOS PARA PROSPECCIÓN MAGNÉTICA DE ESTRUCTURAS ARQUEOLÓGICAS (1)}

\author{
SETTING OF OPTIMAL OPERATIONAL \\ PARAMETERS TO MAGNETICAL \\ PROSPECTING OF ARCHAEOLOGICAL \\ STRUCTURES
}

\author{
JOSÉ ANTONIO PEÑA (*) (**) \\ JOSÉ ANTONIO ESQUIVEL (*) (**) \\ M. OLIVA RODRÍGUEZ-ARIZA (*) \\ BEATRIZ BENJUMEA $(*)(* *)$
}

\section{RESUMEN}

La prospección magnética enfocada a la Arqueología, tiene unas peculiaridades específicas que afectan a la metodología de trabajo y a la operatividad del mismo. En este artículo se evalúan los condicionantes que existen y su influencia en el diseño de la prospección (tratamiento de restos superficiales, espaciado de las estaciones, etcétera) y estudio de las disposiciones óptimas de los sensores en función de las características del terreno. Finalmente, se plantea

(1) Trabajo ligado al Proyecto Sec94-0633 de la CICYT, perteneciente al Plan Nacional de $\mathrm{I}+\mathrm{D}$ y con una duración de tres años. Un resumen de este artículo fue presentado mediante comunicación oral en la VIII Asamblea Nacional de Geodesia y Geofísica, Madrid, 29-V a 1-VI de 1995.

(*) Departamento de Prehistoria y Arqueología, Facultad de Filosofía y Letras, Universidad de Granada. Campus Universitario de Cartuja s/n. 18071 Granada, España.

(**) Instituto Andaluz de Geofísica, Campus Universitario de Cartuja s/n. 18071 Granada. España.

Direcciones e-mail Internet: peruano@ugr.es; esquivel@ululaya.ugr.es; oliva@ululaya.ugr.es; benjumea@ gea.ugr.es

El artículo fue remitido en su versión final el 2-IX-96. una metodología que permite realizar un diseño optimizado de la investigación.

\begin{abstract}
Magnetic exploration applied to Archaeology has some specific characteristics that affect the operability and work methodology. In this article an assessment is made of the existing conditions and their influence on the design of the exploration (e.g. treatment of superficial remains, distances between stations) and the study of optimum positions of the sensors, depending upon the characteristics of the terrain. Finally, a methodology is outlined that permits the accomplishment of an optimized research design.
\end{abstract}

Palabras clave: Arqueología. Magnetismo. Metodología. Parámetros operacionales. Prospección geofísica.

Key words: Archaeology. Magnetism. Methodology. Operational parameters. Geophysical prospecting.

T. P., 53, n. ${ }^{\circ} 2,1996$ 


\section{ANTECEDENTES}

Las publicaciones sobre prospección magnética de estructuras arqueológicas se han ido incrementando a partir de los años 70 , con la disponibilidad de cada vez mejores modelos de magnetómetros portátiles, inicialmente los de saturación de flujo, y posteriormente los de protones y los de bombeo óptico (Telford, Goldart \& Sheriff, 1990). La mayoría de los modelos actuales puede medir el campo magnético local con resolución de una décima o centésima de nanotesla (al menos según sus fabricantes), lo que unido a su portabilidad, facilidad de operación y trasvase de datos a ordenadores, los hacen muy adecuados para el trabajo de campo.

El posterior tratamiento de los datos obtenidos en el terreno ha sido objeto de numerosos desarrollos teóricos, estando en la actualidad muy automatizado, con numerosos programas disponibles, que permiten resaltar en laboratorio los resultados de la prospección. Sin embargo, hay notables discrepancias entre los distintos autores en el planteamiento de la fase de adquisición de datos: temas como el empleo de uno o más sensores y en qué disposición mutua, altura del sensor que actúa como sonda sobre la superficie del terreno, densidad de la rejilla de prospección y tratamiento de los restos arqueológicos superficiales y del material no arqueológico, son resueltos por los distintos autores de maneras muy diferentes y a menudo contradictorias; es más, en muchas ocasiones la elección de un modo de operación u otro no se justifica de manera suficiente.

\section{CONDICIONANTES DE LA PROSPECCIÓN MAGNÉTICA DE ESTRUCTURAS ARQUEOLÓGICAS}

Las estructuras arqueológicas presentan unas características específicas (dimensiones pequeñas, escasa profundidad, geometría, etc.) diferentes de los objetos que se estudian en otros campos como la minería, etc. Estas peculiaridades, que condicionan la metodología a emplear y la interpretación de los resultados, provienen de múltiples factores entre los que pueden destacarse los más importantes:
- Diversidad de tamaño y morfología de estructuras. Los yacimientos arqueológicos al aire libre presentan una gran variedad tipológica: desde la agrupación de varias cabañas en un espacio reducido, hasta ciudades de varias hectáreas de extensión con un urbanismo muy desarrollado donde calles, plazas, edificios públicos y áreas de actividad están separados y son fácilmente identificables. Igualmente, los hombres han creado múltiples ritos funerarios, para el depósito de los muertos o las cenizas (monumentos, fosas, covachas, etc.). Por tanto, esta diversidad en tamaños y morfologías va a condicionar en primer lugar, y según el yacimiento que queremos estudiar, la elección de la rejilla de muestreo, es decir, la distancia entre las estaciones. Ésta habrá de ser menor en yacimientos con estructuras efímeras y en necrópolis, mientras que en poblados o ciudades una mayor distancia entre las estaciones no será un obstáculo para la localización de las grandes estructuras.

- Débil contraste de susceptibilidad magnética entre las estructuras y su entorno. Los materiales del subsuelo ofrecen una respuesta magnética diferente frente a la acción de un campo excitador (campo magnético terrestre), denominada susceptibilidad magnética. En las estructuras arqueológicas es frecuente la utilización de los materiales del entorno (piedras, tierra, etc.) para la realización de las mismas, lo que hace que éstas tengan unas propiedades muy similares a las del substrato del que han sido extraídas. Asimismo, los derrumbes que envuelven a las estructuras son de la misma naturaleza que aquellas de las que proceden, por lo que el contraste de propiedades magnéticas es muy bajo. Por otra parte, los procesos postdeposicionales de tipo erosivo dejan en superficie restos desordenados que se convierten en una fuente de ruido que se añade a las señales que originan las estructuras subyacentes. Esto hace aconsejable el estudio, en un primer momento, del re-

T. P., 53, n. ${ }^{\circ} 2,1996$ 
gistro arqueológico superficial, para definir la naturaleza del asentamiento, a fin de evitar una laboriosa prospección geofísica sobre un yacimiento secundario, y en segundo lugar, sería aconsejable la localización, recogida y catalogación de los restos superficiales para evitar una fuente de perturbación. Es decir, la prospección geofísica se ve facilitada por una previa preparación del terreno a estudiar.

- Existencia de materiales con magnetismo remanente. El origen antrópico de los yacimientos arqueológicos provoca que podamos encontrar metales ferromagnéticos (de acentuada respuesta en presencia de un campo excitador) y materiales termorremanentes (con magnetismo adquirido debido a la acción térmica). Estos últimos pueden clasificarse, desde el punto de vista del interés arqueológico, en dos categorías básicas: los que exigen altas temperaturas en el proceso de elaboración (ladrillos, cerámicas, etc.) y aquellos que han estado sometidos a la acción del fuego de una forma accidental, como consecuencia de grandes incendios. La existencia de alguno o del conjunto de estos materiales permite que, en algunos casos, lleguen a medirse anomalías muy notables, pudiéndose determinar en ocasiones estructuras como hornos y hogares, y áreas de actividades específicas como herrerías y almacenes. En el caso de los metales, una limpieza superficial podrá evitar la perturbación producida por algunos restos férricos modernos presentes en el yacimiento.

- Existencia de superposición de estructuras. La frecuente reutilización del espacio a lo largo del tiempo provoca, en la mayoría de las ocasiones, una reorganización de las estructuras y elementos de construcción, con la destrucción o sepultamiento de las precedentes, lo que puede complicar de manera notable los mapas de anomalías que obtenemos. En general, los mapas de anomalías serán reflejo de las estructuras más superficiales, siempre que se hayan dispuesto de manera adecuada los sensores (distancia entre ellos, altura del sensor inferior, distancia entre las estaciones), cuestiones metodológicas que serán abordadas con más profundidad en un apartado posterior.

\section{ESTRATEGIAS USADAS EN LAS TAREAS DE CAMPO EN PROSPECCIÓN MAGNÉTICA}

Al realizar una prospección con los condicionantes indicados, los diferentes autores proponen (como se indicó anteriormente) distintas estrategias referentes a la preparación del terreno, espaciado horizontal de las estaciones y sistemática de adquisición de datos (número y disposiciones de los sensores).

Con la idea de encontrar las alternativas operativas más adecuadas, se ha realizado una serie de experimentos con un magnetómetro de protones marca Geometrics, modelo G-856X, sobre un asentamiento romano próximo a Las Gabias, Granada. Se pretenden alcanzar unos objetivos metodológicos sobre la prospección magnética de estructuras arqueológicas (que se exponen en el presente trabajo) y unos objetivos arqueológicos que serán detallados en futuras publicaciones. Se quiere responder a las siguientes cuestiones:

- Es o no conveniente una preparación del terreno antes de la prospección magnética.

- Es mejor utilizar uno o dos sensores para la prospección.

- Caso de usar dos sensores, es mejor la medición relativa o la de gradiente.

- A qué altura debe estar el sensor que actúa como sonda.

- Qué distancia debe haber entre sensores caso de medir gradiente.

- Cuál es el espaciado ideal de las estaciones de lectura (Fig. 1).

La serie de experimentos ha consistido en:

- Realización de unos centenares de medidas sobre un mismo punto, tomadas a intervalos temporales regulares, con el fin de encontrar las condiciones reales (no las nominales publicadas por el fabrican- 


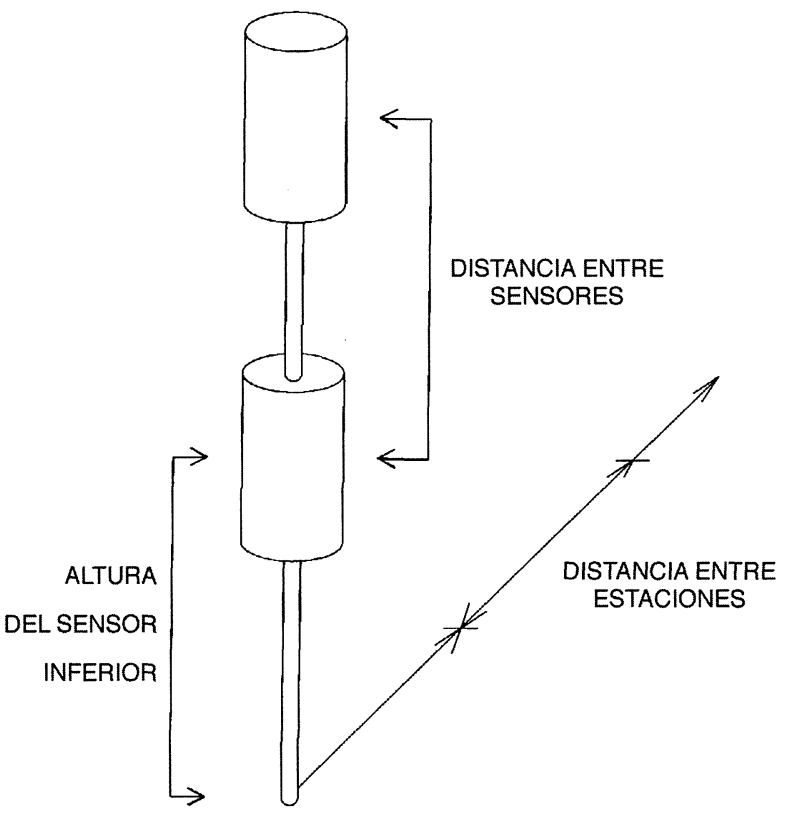

Fig. 1. Muestra una disposición típica de sensores en modo "gradiente" para una prospección magnética de estructuras arqueológicas, los dos sensores están unidos entre sí por un vástago de una longitud determinada. El sensor inferior se apoya a su vez sobre el suelo mediante otro vástago. En el diágrama se indican los parámetros cuyo valor óptimo se desea determinar:

- Distancia entre los sensores.

- Altura del sensor inferior sobre la superficie del terreno.

- Distancias entre los sucesivos nodos de la rejilla.

te) de resolución del magnetómetro actuando con dos sensores y una única consola.

- Visitas al terreno para obtener información sobre la geometría de las estructuras capaces de producir anomalías detectables por métodos magnéticos.

- Simulación numérica de las anomalías obtenidas en método sensor único o medición relativa y en modo gradiente.

- Simulación numérica de diferentes distancias entre sensores en modo gradiente.

- Cálculo de las longitudes de onda de las anomalías originadas por las estructuras observadas y determinación de la frecuencia espacial de muestreo óptima para representar tales anomalías de una manera adecuada.
- Realización de perfiles a lo largo de una anomalía, con diferentes alturas del sensor inferior y en diferentes condiciones de preparación del terreno, a fin de encontrar la combinación más favorable.

A continuación, se revisan las soluciones propuestas por los diferentes autores, a cada uno de los interrogantes planteados y se indican nuestras aportaciones, basadas en la serie de experiencias realizadas.

\section{ESTAQUILLADO DEL TERRENO}

Suele realizarse antes de la prospección, en ocasiones con levantamiento simultáneo del mapa topográfico mediante estaciones totales (Romero Sanz, 1995; Cole, Cottrell \& Payne, 1995), para permitir la toma de lecturas de manera sistemática.

El sistema adoptado en este estudio ha consistido en la realización de un estaquillado (se ha aplicado al ya citado yacimiento de Las Gabias), usando medios topográficos convencionales, con división del terreno en cuadrados de una superficie de un área cada uno y con rumbos N-10-E y N-100-E. Posteriormente, se ha realizado un levantamiento topográfico detallado (escala 1:500) mediante estación total, incluyendo en el mismo el cuadriculado de prospección.

La elección de este tamaño de cuadrícula de referencia es por su facilidad de prospección en un tiempo razonable (una hora con alta densidad del muestreo) y por el gran número de medidas redundantes que permiten un estricto control de la calidad de las lecturas (los perfiles se realizan N-S y S-N, de la cuadrícula, con los bordes de los cuadrados por duplicado), con el valor añadido de la facilidad que supone para cualquier cálculo el tener una longitud característica $\mathbf{x}$ de $10 \mathrm{~m}$.

\section{ELIMINACIÓN DE ELEMENTOS PERTURBADORES MODERNOS}

Algunos autores (OCSA, 1993) recomiendan el uso de detectores de metales para la localización de la basura férrica que deberá ser eliminada antes de la prospección. 
Por otro lado, dado que se trata de yacimientos arqueológicos, es normal que el lugar haya recibido ya las atenciones de otros arqueólogos, así que en algunos casos estará sembrado de pequeños objetos metálicos (chapas de bebidas y similares) arrojados ex profeso como medida de protección para confundir a los detectores de metales de los furtivos, pero que también introducen ruido en las lecturas de los magnetómetros (aunque los materiales no magnéticos son indetectables por el magnetómetro, p.e., el aluminio).

$\mathrm{Si}$ el yacimiento fue parcialmente excavado en época pretérita, el problema es aún más agudo ya que a pesar de que hayan desaparecido las trazas de las excavaciones o "catas" realizadas, es frecuente que queden los clavos del sistema de referencia utilizado, clavos de notable tamaño fijados concienzudamente a distancias constantes y muy bien alineados en direcciones N-S y E-W, susceptibles de originar anomalías muy llamativas.

En el asentamiento estudiado se ha usado el detector de metales para la localización de material férrico, comprobándose la notable conveniencia de esta práctica; se han encontrado herraduras, restos de aperos de labranza, chatarra de automóviles y otra variedad de objetos capaces de producir sus propias anomalías y enmascarar las que interesan.

\section{TRATAMIENTO DE LOS ARTEFACTOS Y RESTOS ESTRUCTURALES DISPERSOS}

Son escasas en la bibliografía las referencias al tratamiento dado a los restos de artefactos, ecofactos y elementos estructurales esparcidos por la superficie debido a procesos postdeposicionales de tipo erosivo. La idea que subyace es que estos restos no deben ser tocados para no perturbar la información que puedan proporcionar, en la idea de que la prospección geofísica será previa a cualquiera otra intervención.

Sin embargo, en algunos casos estos restos, aun proporcionando una notable información cultural acerca de los materiales infrayacentes, producen escasa información espacial, ya que han sido desplazados por las labores agrícolas durante cientos o miles de años.
Este material es una fuente de ruido que se añade a las señales originadas por las estructuras subyacentes. En cualquier caso, la distribución de dichos restos debe ser estudiada previamente aunque sea de manera somera, a fin de evitar la realización de una laboriosa prospección geofísica sobre un yacimiento secundario.

Es deseable que estos restos superficiales sean recogidos y catalogados en prospecciones arqueológicas superficiales previas a la excavación (éste debe ser el procedimiento normal). Por tanto, una solución correcta consiste en realizar la prospección magnética con posterioridad a la prospección arqueológica superficial, para eliminar una fuente de perturbación y conseguir algunas indicaciones acerca del material que puede existir bajo la superficie. Cuando la prospección magnética se realice, la superficie debe estar exenta de elementos perturbadores.

Si esto no fuera posible, será necesario elevar la altura del sensor, en una cuantía que habrá de determinarse, de modo que los restos superficiales no influyan en las lecturas. Normalmente, esta elevación del sensor implicará una cierta pérdida de información sobre la capa más superficial.

\section{ESPACIADO HORIZONTAL DE LAS ESTACIONES}

Este tema es abordado por la mayoría de los autores, que tratan de llegar a un compromiso entre lo deseable (las estructuras buscadas son poco profundas y generalmente de dimensiones métricas, y a menor distancia entre estaciones mejor es el muestreo) y lo razonable (con estaciones separadas $1 \mathrm{~m}$. hay que realizar 10.000 medidas por hectárea, extensión de bastantes yacimientos, mientras que si la separación es de $0,5 \mathrm{~m}$. el número de medidas se eleva a 40.000 ; dos semanas de trabajo de campo en el primer caso y dos meses en el segundo...).

Muchos autores optan por una distancia uniforme (normalmente de $1 \mathrm{~m}$., ocasionalmente 0,5 m.) (Becker, 1978; Martin, 1978; Papamarinopoulos, 1986; Telford, 1990; Hernández, 1992; Sanz Núñez, 1992; OCSA, 1993). Los investigadores de la Historic Buildings \& Monuments 
Commission for England, que realizan numerosos trabajos de gran extensión superficial, usan de manera sistemática desde 1992 perfiles N-S separados por una distancia de $1 \mathrm{~m}$. y dentro de cada perfil toman las lecturas cada $25 \mathrm{~cm}$. (AAVV, 1992-1995). Otros prefieren usar una rejilla de prospección uniforme (normalmente mayor) para localizar las anomalías generales y otra dentro de ésta, de mayor densidad espacial, para delimitar mejor las anomalías detectadas por la primera red (Aitken, 1974; Tsokas, 1986; Casas, 1991; García, 1992).

Una forma de abordar el problema consiste en fijar el espaciado de la rejilla de prospección a partir del conocimiento de la longitud de onda mínima que se espera produzcan las anomalías a prospectar, si ésta es conocida se puede aplicar el Teorema del Muestreo de Shanon, que permite calcular, en nuestro caso, la distancia máxima entre muestras para representar adecuadamente cierta longitud de onda. Este razonamiento ha sido aplicado recientemente por algún autor (Romero Sanz, 1995) que ha empleado un desarrollo del Teorema de Shanon realizado por Keating (1992) para la prospección gravimétrica.

En esencia, el Teorema del Muestreo (Båth, 1974) expresa que para que una onda sea adecuadamente representada mediante un muestreo discreto igualmente espaciado, la frecuencia del muestreo ha de ser, al menos, el doble de la frecuencia de la onda muestreada. Es decir hay que tomar dos muestras, como mínimo, en un ciclo completo para que a partir de ellas se pueda reconstruir la onda original. Puesto que esto es válido, tanto para frecuencia temporal como para frecuencia espacial, quiere decir que la distancia máxima entre muestras ha de ser igual a una semilongitud de la onda que se pretende muestrear.

La mínima longitud de onda esperada se puede calcular si existe un cierto conocimiento del tamaño y profundidad de las estructuras que cabe esperar, lo que suele conseguirse con un adecuado reconocimiento del terreno, en este caso se puede modelizar la perturbación producida por la estructura esperada, con algún programa como MAGPOLY (GEOSOFT, 1985) y calcular posteriormente $\lambda$ (mediante la transformada rápida de Fourier $F F T$, por ejemplo).
Así, aplicando este método para el caso estudiado, que es adaptable a gran número de yacimientos: muros de $0,5 \mathrm{~m}$. de anchura, enterrados a $0,5 \mathrm{~m}$. de profundidad, con el campo total, declinación e inclinación de nuestras latitudes y con el sensor a $0,5 \mathrm{~m}$. de altura, resultan longitudes de onda de unos 6,6 m., lo que implica, de acuerdo con el Teorema de Shanon, que se pueden muestrear perfectamente las anomalías tomando lecturas a una distancia horizontal de 3,3 m. Por tanto, la distancia de $1 \mathrm{~m}$. tomada como estándar por muchos autores es más que suficiente; en realidad, origina un notable sobremuestreo (lo que no es totalmente malo, si hay tiempo y fondos), que permite detectar longitudes de onda menores (hasta $2 \mathrm{~m}$.) producidas por estructuras más pequeñas o muy próximas a la superficie. Distancias mayores que $3 \mathrm{~m}$. sólo deben tomarse si el cuerpo productor de la anomalía es más profundo y/o si el sensor se coloca a mayor altura; distancias de muestreo inferiores al metro $(0,5 \mathrm{~m}$. es también empleada de modo frecuente) permiten, por supuesto, muestrear mejor la señal, pero no proporcionan más información y en contrapartida, son notablemente más costosas.

En el caso de tomar lecturas en modo gradiente, la longitud de onda resultante en iguales condiciones que las anteriores y con una separación entre sensores de $1 \mathrm{~m}$., resulta ser de $5 \mathrm{~m}$., lo que implica, de acuerdo con el Teorema antes citado, la necesidad de muestrear con una distancia horizontal de 2,5 m. De este modo un muestreo con distancia de $2 \mathrm{~m}$. entre las estaciones será satisfactorio. La técnica usual de muestrear a $1 \mathrm{~m}$. es también es admisible, ya que describe mejor la señal y permite la detección de anomalías producidas por estructuras más pequeñas o superficiales no previstas inicialmente.

\section{ELECCIÓN DEL NÚMERO DE SENSORES Y DEL MÉTODO DE ADQUISICIÓN}

Es tratada de diferentes maneras por los distintos autores siendo las estrategias más usuales las siguientes:

1. Medidas con un solo sensor y corrección temporal mediante puntos de control, que pueden ser puntos cualesquiera de la rejilla, sobre 
los que se realizan medidas redundantes o bien medidas repetidas cada cierto lapso de tiempo en un mismo punto que se toma como referencia. Es el método empleado cuando se dispone de un solo sensor (Sanz Núñez, 1992).

2. Medida diferencial mediante un magnetómetro fijo que actúa como referencia y otro móvil que actúa como sonda. Es un método usual cuando se dispone de dos sensores y una sola consola (los dos sensores están unidos a la consola mediante un cable de longitud suficiente y son disparados sucesivamente con intervalo de escasos segundos). Cuando se dispone de dos magnetómetros completos, el magnetómetro que está en estación toma lecturas a intervalos regulares. Este último método es muy interesante al permitir programar la sonda móvil

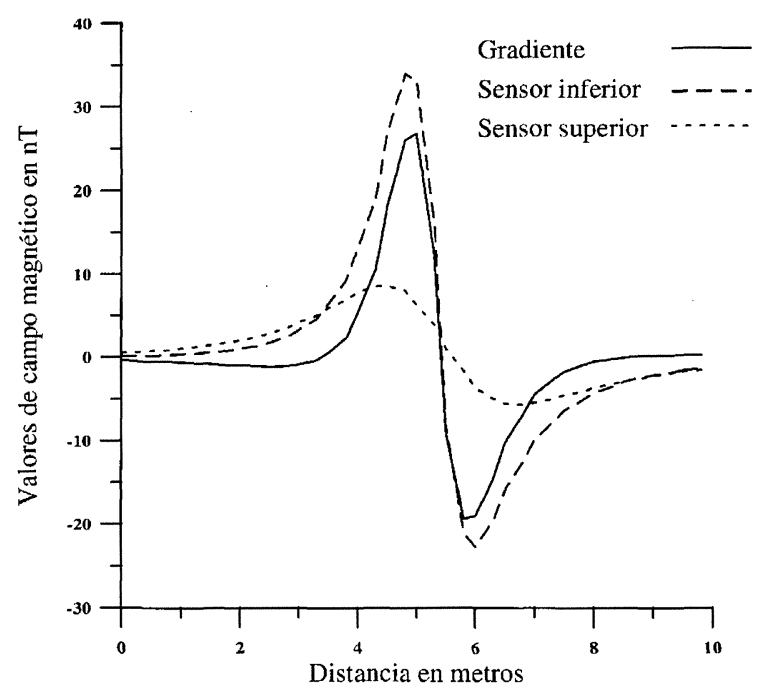

Fig. 2. Comparación entre gradiente y lecturas de sensor único.

Muestra tres curvas obtenidas por simulación numérica para un paralelepípedo de $0,5 \times 1 \mathrm{~m}$. situado entre los 5 y 5,5 , enterrado a $0,5 \mathrm{~m}$. de profundidad (las condiciones de magnetismo reinantes en nuestra región son $55^{\circ}$ de inclinación, 43.000 nanoteslas de campo externo y contraste de susceptibilidades de $10^{-3}$ ).

La curva para el sensor superior (situado a $1 \mathrm{~m}$. de altura) muestra una anomalía amplia, de extensión superior al tamaño del cuerpo productor; la curva para el sensor situado sobre el terreno muestra la anomalía mejor adaptada al cuerpo productor; la curva de gradiente está aún más ajustada (los picos casi coinciden con los límites $\mathrm{S}$ y $\mathrm{N}$ del cuerpo anómalo), aunque la amplitud medida es algo menor.

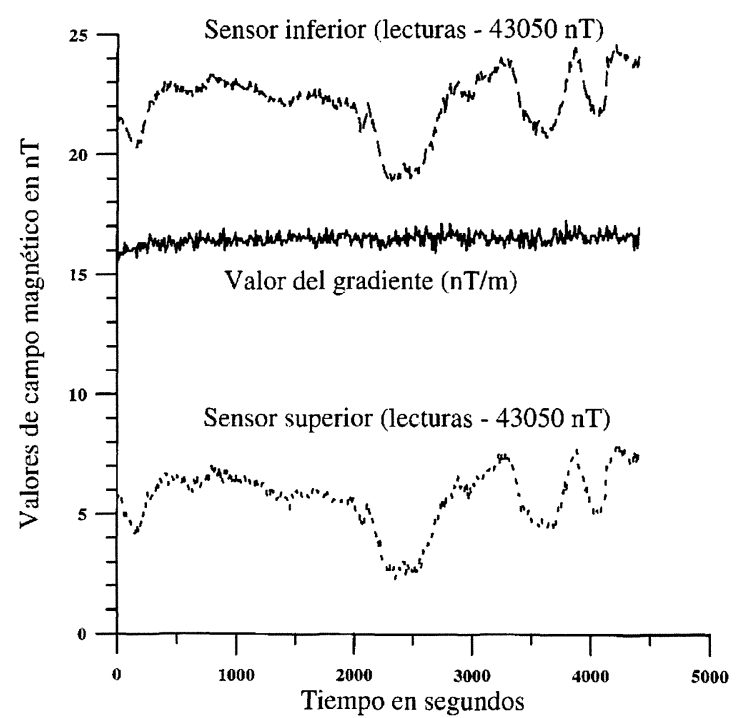

Fig. 3. Determinación de la resolución.

El método de "gradiente" exige que los dos sensores se disparen en rápida sucesión, impidiendo aprovechar la máxima resolución nominal del magnetómetro (una mera cuestión instrumental). La realización de numerosas medidas en condiciones estacionarias muestra que la resolución, en estas condiciones es del orden de 1 nanotesla por metro.

para una resolución máxima y poder eliminar eficientemente la variación diurna, mientras que si los dos sensores son disparados por la misma consola no se aprovecha la resolución máxima (por simples exigencias del aparataje), aunque la diferencia de tiempos entre las lecturas de los sensores sea mejor.

Experiencias con algunas de estas modalidades de magnetómetro diferencial han sido realizadas por numerosos autores (Becker, 1978; Martin, 1978; Weymouth, 1978; Tsokas, 1986; Hernández, 1992).

3. Medida de "gradiente" mediante dos sensores situados en la misma vertical.

Este último método suele ser el preferido mayoritariamente en la actualidad (Tsokas, 1986; Telford, 1990; Sanz Núñez, 1992; OCSA, 1993; AAVV 1992-1995) y es el usado en el presente trabajo, toda vez que evita la corrección temporal y atenúa las anomalías regionales a favor de las más locales y superficiales, midiendo de este modo unas anomalías bastante ajustadas al cuerpo productor (Fig. 2), de hecho la 
longitud de onda del gradiente es menor que la longitud de onda del sensor inferior.

Como contrapartida obliga a ajustar los switches (microinterruptores de control de las funciones internas) del aparato para disparos sucesivos en el intervalo de pocos segundos, lo que impide aprovechar la máxima resolución nominal del mismo, como lo demuestra la realización previa de lecturas en condiciones estáticas para determinar la resolución real del magnetómetro (un modelo Geometrics de protones) actuando en estimación de gradiente, lo que permite concluir que su resolución en condiciones operativas es de 1 nanotesla, bastante lejos de las 0,1 nanoteslas de resolución nominal (Fig. 3).

\section{DISTANCIA ENTRE LOS SENSORES Y ALTURA DEL SENSOR INFERIOR EN EL MÉTODO DEL GRADIENTE}

Otros puntos de discrepancia entre los diferentes investigadores lo constituyen caso de medir "gradiente" la distancia entre los sensores y en cualquier caso, la altura de la sonda sobre el suelo. En cuanto al primero hay tres tendencias y se procura que la distancia sea:

- La mínima posible, a fin de acercarse al ideal matemático de que sea un infinitésimo de la profundidad del objeto productor de la anomalía, tesis sostenida por los más puristas (Breiner, 1973; Sanz Núñez, 1992).

- La máxima prácticamente admisible, a fin de maximizar la diferencia, tesis defendida por los más pragmáticos (Breiner, 1973; Tsokas, 1986).

- Una distancia intermedia, solución adoptada por los más eclécticos (Telford, 1990; OCSA, 1993).

Las experiencias que hemos realizado tanto por modelización, usando diferentes distancias entre sensores, como por lecturas sobre el terreno, también con diferentes separaciones entre los sensores, demuestran que para un contraste de susceptibilidades "normal" la distancia entre los sensores no es determinante, obteniéndose gráficas de lecturas de gradiente muy similares, excepto cuando el sensor infe- rior está demasiado alto (a más de $1 \mathrm{~m}$. de altura) en el que la curva de anomalía aparece muy poco marcada (Fig. 4).

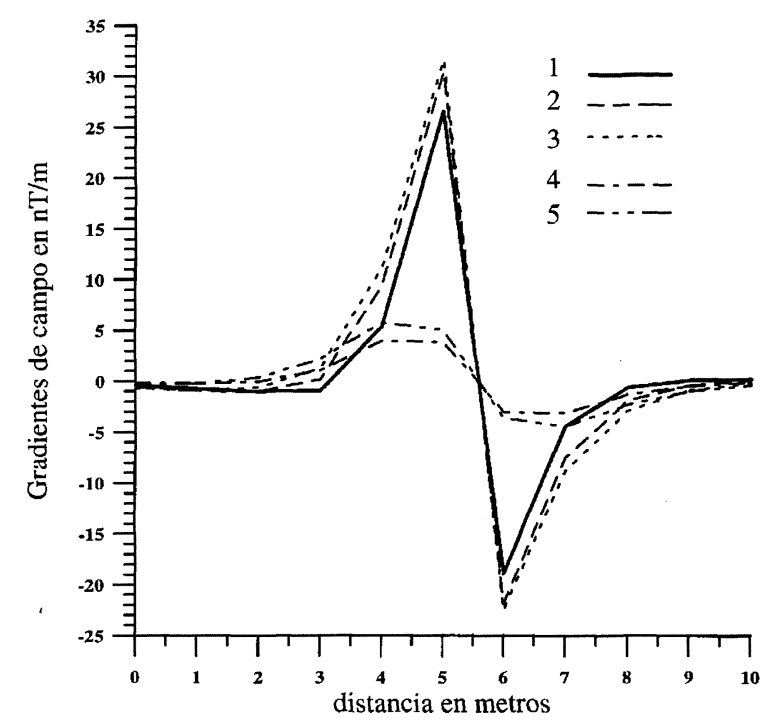

Fig. 4. Simulación numérica de lecturas con un solo sensor mediante perfiles sobre un cuerpo enterrado (las mismas condiciones que en la figura 2). Se utilizan diversas combinaciones de distancias entre sensores y altura del sensor inferior sobre la superficie (en 1, 2 y 3 el sensor inferior está a $0 \mathrm{~m}$. sobre el terreno y el superior a 1,2 y 3 , respectivamente; en 4 y 5 el sensor inferior está a $1 \mathrm{~m}$. de altura sobre el terreno y el superior a 2 y 3 , respectivamente). Se observa que la distancia entre sensores no es relevante cuando el sensor inferior está muy cercano a la superficie.

El tema de la altura sobre el terreno del sensor que actúa como sonda es tratado de distinto modo por los diferentes autores, con dos opciones principales:

- La sonda lo más cerca posible del terreno, a fin de obtener la máxima señal, aun a costa (si se baja demasiado) de incluir un notable ruido. Así, entre las opciones más utilizadas, aparecen: bajo (Breiner, 1973); no menor de 0,3 m. (Aitken, 1974); a muy pocos cm. (OCSA, 1993); sobre el terreno (Casas, 1991); próximo al suelo (García, 1992); $50 \mathrm{~cm}$. (Hernández, 1992); a unos $30 \mathrm{~cm}$. del suelo (Sanz Núñez, 1992).

- La sonda a bastante altura sobre el suelo, minimizando el ruido y aprove- 


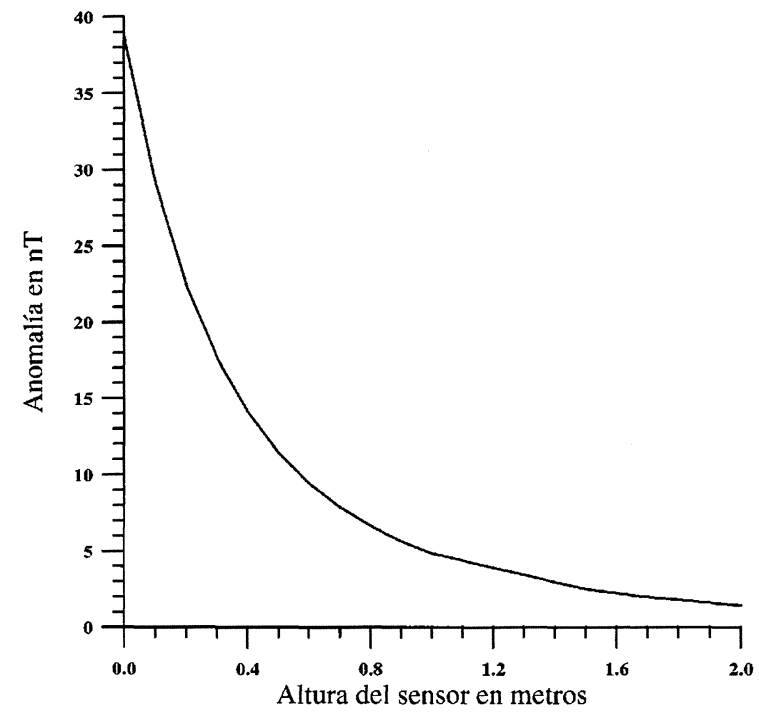

Fig. 5. Variación de la anomalía con la altura de un solo sensor. Simulación numérica que muestra la disminución de la señal en función inversa con la altura del sensor (el cuerpo anómalo es un cubo de $1 \mathrm{~m}$. de arista enterrado a $0,5 \mathrm{~m}$. de profundidad). En las mismas condiciones de entorno que en otras simulaciones anteriores. Con un sensor sobre la superficie la intensidad de la señal sería lo suficiente para saturar el magnetómetro; a $2 \mathrm{~m}$. de altura la señal es muy débil y próxima al límite de resolución del aparato en las condiciones operacionales.

chando la máxima resolución nominal del magnetómetro; Tsokas (1986) aconseja colocarlo a $1,5 \mathrm{~m}$.

En el yacimiento en estudio se ha optado por realizar algunas visitas al lugar a prospectar para hacer observaciones tendentes a determinar, en afloramientos cercanos, algunas morfologías, tamaños y profundidades de las estructuras.

A continuación, se han realizado los cálculos, basados en las condiciones deducidas del paso anterior, de la variación de la señal con la altura para un cubo de $1 \mathrm{~m}$. de arista enterrado a $0,5 \mathrm{~m}$. de profundidad, puede comprobarse que se obtienen señales muy grandes a nivel del suelo y cercanas al límite de resolución del aparato a unos $2 \mathrm{~m}$. de altura (Fig. 5).

$$
\mathrm{T}=\frac{\mathrm{KFD}^{3}}{\mathrm{r}^{3}}
$$

donde: $\mathrm{T}=$ intensidad de campo

$\mathrm{F}$ = valor del campo inductor

$\mathrm{K}$ = susceptibilidad magnética

$\mathrm{D}=$ longitud de la arista del cubo

$\mathrm{r}=$ distancia desde el centro del cubo a la sonda

Esto sugiere, en principio, que para estructuras arqueológicas, enterradas a poca profundidad, el sensor ha de estar "bajo". Con el objeto de cuantificar esta altura mínima se ha realizado sobre el terreno una serie de lecturas sobre un mismo perfil, a través de una anomalía detectada en una exploración previa, usando cinco alturas diferentes del sensor inferior. La comparación de los perfiles realizados permite comprobar que éstos se unifican (en este caso concreto) a partir de $48 \mathrm{~cm}$. de altura. Para alturas superiores el rango de la anomalía disminuye acercándose a los límites de resolución del gradiómetro. Este método, resumido en la figura 6 , permite la determinación experimental de la altura óptima del sensor inferior para un asentamiento determinado.

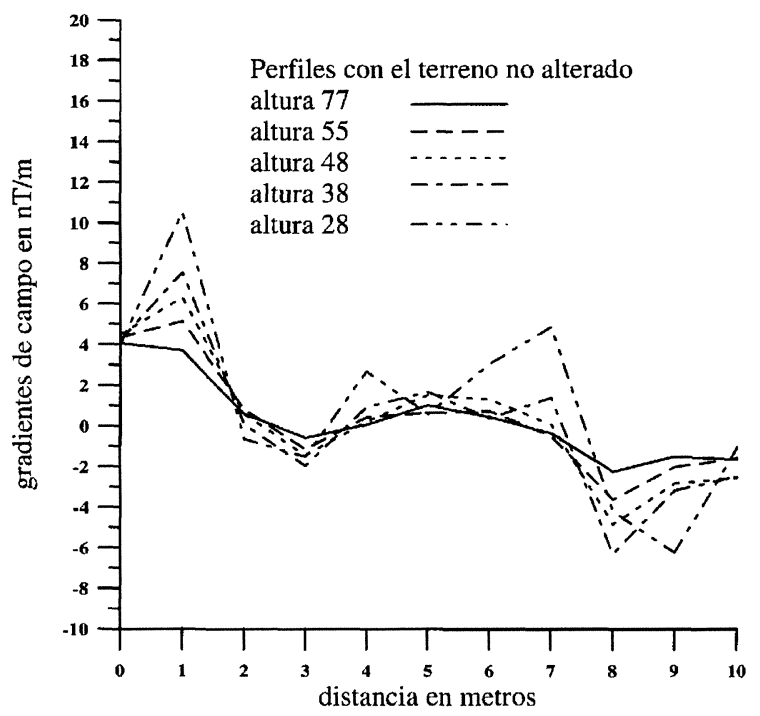

Fig. 6. Realización de perfiles a lo largo de una anomalía detectada; se han hecho con diferentes alturas del sensor inferior y con una distancia constante entre sensores. A menor altura aparecen discrepancias producidas por elementos sueltos (en superficie o soterrados) cercanos al sensor. A partir del medio m. de altura se atenúa el ruido. 


\section{CONCLUSIONES}

Como se ha indicado a lo largo del texto, la prospección magnética de estructuras arqueológicas presenta una serie de peculiaridades que tienen bastante influencia en los resultados obtenidos. En este trabajo se han analizado estas características, obteniéndose las siguientes conclusiones aplicables a asentamientos con bajo ruido antrópico:

1. La determinación del espaciado horizontal debe realizarse en función del tipo de anomalías esperadas, teniendo en cuenta la longitud de onda de dichas anomalías. En este sentido, la decisión más operativa consiste en un compromiso entre lo deseable y lo posible, teniendo como directriz el Teorema de Shanon (p. e., el aumento de la frecuencia de muestreo no conduce automáticamente a una mejora en la discriminación, sino que está limitada por factores como la profundidad de la estructura buscada, distancia del sensor a la superficie, etc.).

2. Es necesario llevar a cabo la recolección previa de los elementos perturbadores dispersos por la superficie (metales férricos, materiales con magnetismo termorremanente, etc.), que influyen en las mediciones. En su defecto puede colocarse el sensor a mayor altura, detectando únicamente las anomalías más importantes.

3. En el método de estimación de gradiente, la resolución real no suele coincidir con la resolución nominal del dispositivo. Así, es recomendable la realización de pruebas en condiciones estáticas para determinar la resolución real del magnetómetro.

4. Aunque existen distintas disposiciones de los sensores, el método de "gradiente" proporciona buenos resultados con un mínimo de complicaciones. Evita las correcciones temporales, atenúa las anomalías regionales y resalta las locales, que quedan muy ajustadas a la situación del cuerpo productor.

5. La distancia del sensor inferior al suelo debe ser la menor posible, para detectar las anomalías producidas por las estructuras pequeñas y suficientemente grande para minimizar el ruido producido por los restos sueltos incluidos en las capas más superficiales. La altura óptima debe ser determinada de forma experimental en el propio asentamiento, según el método propuesto en la figura 6 .

6. La distancia entre los sensores no es determinante en lugares donde el contraste de susceptibilidades es "normal".

La aplicación de estas recomendaciones permite un diseño de prospección bastante operativo, consiguiendo una buena relación señal-ruido y una adquisición de datos fiable y económica, al evitar sobremuestreos incontrolados.

\section{BIBLIOGRAFÍA}

AAVV (1992-1995): “Ancient Monuments Laboratory Reports on Geophysical surveys". Historic Buildings \& Monuments Commission for England, Internet, http://robin.eng-h.gov.uk/reports.

Aitken, M.J. (1974): Physics and Archaeology. Oxford University Press. Oxford.

BÅTH, M. (1974): Spectral Analysis in Geophysics. Elsevier. Amsterdam.

BECKER, H. (1978): "Magnetic prospecting with a difference-protonmagnetometer with an automatic data-record on a digital cassette". Proceedings of the 18th International Symposium on Archaeometry and Archaeological Prospection (Bonn, 14-17 March 1978). Landschafsverband Rheinland Rheimisches Landesmuseum. Bonn: 633-636.

BREINER, S. (1973): Applications manual for portable magnetometers. Geometrics. Sunnyvale.

Casas, A.; Pinto, V.; Gurt, J.M.; Riera, S. \& Burés, L. (1991): "Magnetic survey and data processing applied to the location of roman kilns: methodological evaluation and archaeological results”. En P. Budd, B. Chapman, C. Jackson, R. Janaway \& B. Ottaway (eds.): $A r$ chaeological Sciences 1989. Oxbow Monography 9. Proceedings of a conference on the application of scientific techniques to archaeology (Bradford, September 1989): 265-272.

Cole, M.; Cottrell, P. \& Payne, A. (1995): “Old Winchester Hill, Corhampton, Hampshire. Preliminary Report on Geophysical Survey, February 1995. Ancient Monuments Laboratory report". Historic Buildings \& Monuments Commission for England Internet, http://www.eng-h.gov.uk/reports/oldwinch.

GarCíA, A. y DíEZ, J.L. (1992): "Magnetometría en prospección arqueológica". Jornadas sobre teledetección y geofísica aplicadas a la arqueología (Madrid, 1986): 4347.

GEOSOFT (1985): Programa de ordenador MAGPOLY 2.13 .

Hernández, M.C.; Cámara, m.C.; Velasco, F.; Mena, P. y Gras, R. (1992): “Aplicación de algunos métodos geofísicos (eléctrico, magnético y gravimétrico) en

T. P., 53, n. ${ }^{\circ} 2,1996$ 
Fosos de Bayona (Huete, Cuenca)". Jornadas sobre teledetección y geofísica aplicadas a la arqueología (Madrid, 1986). Ministerio de Cultura. Instituto de Conservación y Restauración de Bienes Culturales. Madrid: $85-92$.

Hernández, M.C.; Cámara, M.C.; Mena, P. y Nogueras, E. (1992): "Prospección magnética realizada en la necrópolis de El Navazo (Cuenca) y resultados arqueológicos". Jornadas sobre teledetección y geofísica aplicadas a la arqueología (Madrid, 1986). Ministerio de Cultura. Instituto de Conservación y Restauración de Bienes Culturales. Madrid: 93-98.

Keating, P. (1992): "Determination of the optimum gridding interval of a gravity data set". 54th Meeting \& Technical exhibition of the European Association of Exploration Geophysicists (Paris, 1992): 378-379.

MARTin, A.; MunRo, R. \& PAPAMARINOPOUlos, S. (1978): "The investigation of an unusual magnetic anomaly by combined magnetometer and soil susceptibility surveys". Proceedings of the 18th International Symposium on Archaeometry and Archaeological Prospection (Bonn, 14-17 March 1978). Landschafsverband Rheinland Rheimisches Landesmuseum. Bonn: 675-680.

OCSA (1993): Reconocimiento arqueofísico en Baelo Claudia, Cádiz. Junta de Andalucía. Consejería de Cultura y Medio Ambiente. Sevilla.

Papamarinopoulos, S.P.; Tsokas, G. \& Williams, H. (1986): "Magnetic and electric measurements in the Island of Lesbos". En Y. Liritzis, \& T. Hackens (eds.): Abstracts of First South European Conference in Archaeometry 1984 (Delphi, 1984). Louvain-la-Neuve.

PeÑa J.A.; Esquivel, J.A.; Rodríguez-ArizA, O. y BenJUMEA, B. (1995): "Establecimiento de la altura óptima del sensor para prospección magnética de estructuras arqueológicas". Programa y Resúmenes de la VIII Asamblea Nacional de Geodesia y Geofísica (Madrid,
1995). Instituto Geográfico Nacional y Universidad Complutense de Madrid. Madrid: 154.

Romero SANZ, A. (1995): Prospección magnética en áreas de intenso ruido cono herramienta de análisis en la ingeniería civil. Aplicación conjunta con técnicas de georradar a la zona de sincrotón de Cataluña. Projecte o Tesina d'Especialitat, E.T.S. de Ingenieros de Caminos, Canales y Puertos de Barcelona.

SANZ NúNEEZ, A.C. (1992): "Aplicación de magnetómetros de protones en prospecciones arqueológicas. Ventajas e inconvenientes". Jornadas sobre teledetección y geofísica aplicadas a la arqueología (Madrid, 1986). Ministerio de Cultura. Instituto de Conservación y Restauración de Bienes Culturales. Madrid: 37-41.

Telford, W.; Goldart, L.P. \& SHERIFF, R.E. (1990): Applied Geophysics, second edition. Cambridge University Press. Cambridge.

Tsokas, G.N.; Rocca, A.C. \& Papazachos, B.C. (1986): "Magnetic prospecting at the prehistoric site of the Village of Mandalo in Northern Greece". En Y. Liritzis \& T. Hackens (eds.): Proceedings of First South European Conference in Archaeometry 1984 (Delphi, 1984). Louvain-la-Neuve: 143-151.

TsoKas, G.N.; RocCA, A.C. \& PAPAZaChos, B.C. (1986): "Some aspects concerning the application of geophysical prospecting methods at the Dion Archaeological Site". En Y. Liritzis \& T. Hackens (eds.): Proceedings of First South European Conference in Archaeometry 1984 (Delphi, 1984). Louvain-la-Neuve: 153-161.

WEYMOUTH, J. (1978): "Tecnical developments and results of the 1977 season of the Great Plains magnetic surveying program". Proceedings of the 18th International Symposium on Archaeometry and Archaeological Prospection (Bonn, 14-17 March 1978). Landschafsverband Rheinland Rheimisches Landesmuseum. Bonn: 710-717. 